\title{
Dynamic rupture propagation on geometrically complex fault with along-strike variation of fault maturity: insights from the 2014 Northern Nagano earthquake
}

\author{
Ryosuke Ando ${ }^{1 *}$, Kazutoshi Imanishi ${ }^{2}$, Yannis Panayotopoulos ${ }^{3,5}$ and Tomokazu Kobayashi ${ }^{4}$
}

\begin{abstract}
Understanding the effect of the complex fault geometry on the dynamic rupture process and discriminating it from the complexity originating from the rheological properties of faults, is an essential subject in earthquake science. The 2014 Northern Nagano earthquake, which occurred near the end zone of a major active fault system, provided unique observations that enabled us to investigate both the detailed geometrical fault structure and surface deformation patterns as well as the temporal sequence led up from a prominent foreshock activity. We first develop a geometrical fault model with a substantial variation along strike, and a model for the regional stress field, which is well constrained by the observations. This significant along-strike variation in fault geometry probably reflects the difference of fault maturity at the end zone of the complex fault system. We used this model in order to conduct a set of dynamic rupture simulations using the highly efficient spatiotemporal boundary integral equation method. Based on our simulations, we show that the observed surface deformation can be reasonably explained as the effect of the non-planar fault geometry with a number of branch faults and bends.
\end{abstract}

Keywords: 2014 Northern Nagano earthquake, Fault geometry, Dynamic rupture, InSAR, Fast domain partitioning BIEM

\section{Introduction}

The geometry of natural fault surfaces tends to become smoother and simpler as the cumulative slip becomes larger (e.g., Wesnousky 1988). Moreover, when tectonic stressing conditions are changed, fault geometries start to evolve in order to adapt to the new stress conditions (e.g., Scholz et al. 2010). The Itoigawa-Shizuoka tectonic line (ISTL) shown in Fig. 1 is one of the major active inland fault systems, which experienced such a change in the tectonic conditions; the ISTL intersects the central part of the Honshu island and forms a 150-km-long boundary

\footnotetext{
*Correspondence: ando@eps.s.u-tokyo.ac.jp

1 Department of Earth and Planetary Science, School of Science, University of Tokyo, 7-3-1 Hongo, Bunkyo-ku, Tokyo 113-0033, Japan Full list of author information is available at the end of the article
}

between the northeastern and southwestern Japanese Honshu island arc. The ISTL defines the western margin of the Fossa Magna rift basin developed under a tensional stress field associated with the final stages of the opening of the Sea of Japan (25-15 Ma) (Otofuji et al. 1985; Yamaji 1990). Later, the basin is reactivated under the currently dominant east-west compressional stress field exhibiting shortening deformation since $3 \mathrm{Ma}$ (e.g., Sato 1994). At the present stage, based on geomorphological appearance (Kato et al. 1989; Nakata and Imaizumi 2002; Matsuta et al. 2004; Kondo et al. 2008), the northern end of the ISTL is considered currently inactive as roughly illustrated in Fig. 1.

The 2014 Northern Nagano earthquake occurred at a unique site near the northern end section of the currently active ISTL. It occurred on November 22 at local 


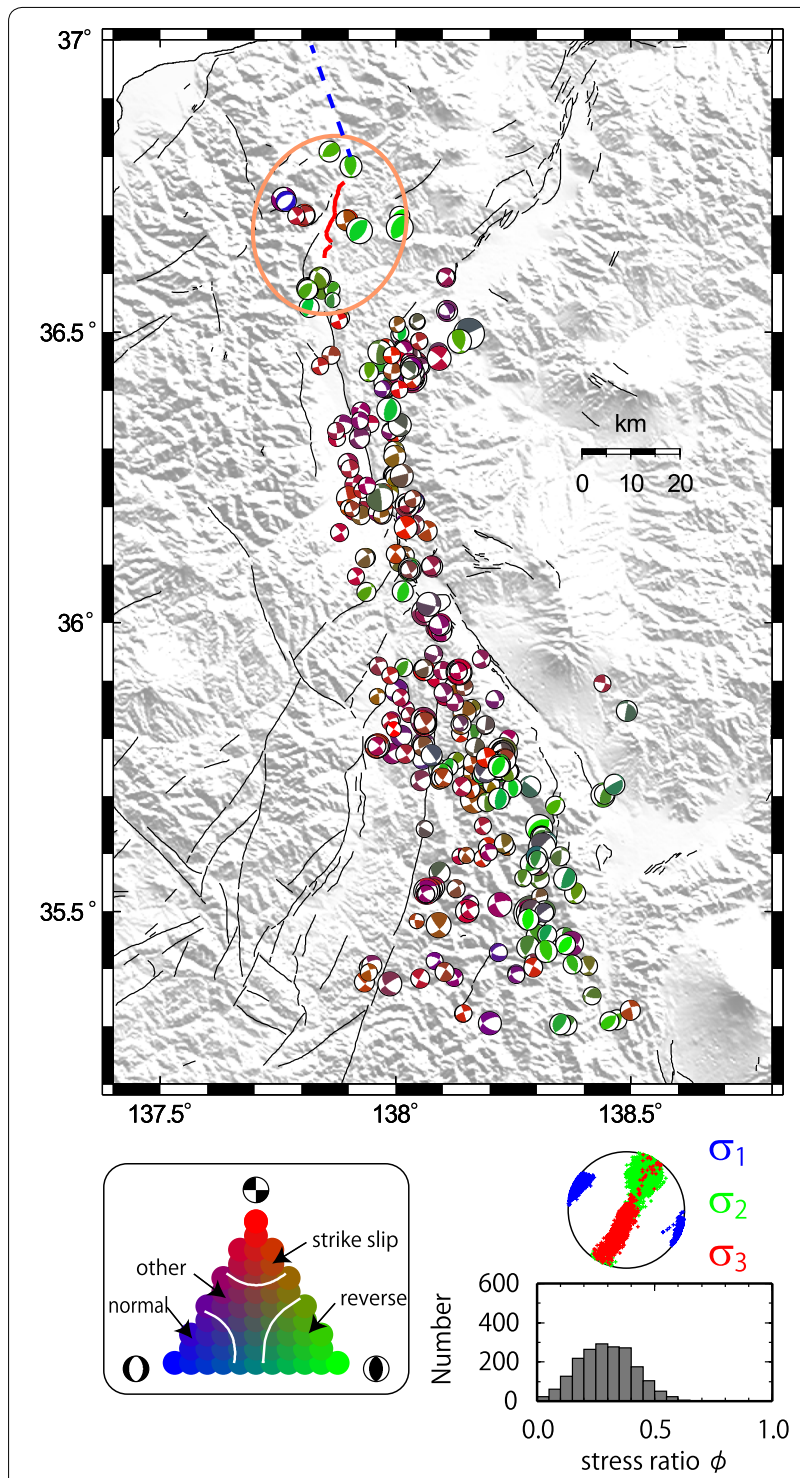

Fig. 1 Observed focal mechanisms and principal stress state obtained before the 2014 Northern Nagano earthquake. The map view shows the focal mechanisms picked along the ItoigawaShizuoka tectonic line (ISTL) (see the legend for colored indicating the faulting types); the black lines denote the active fault traces, and the Kamishiro fault is highlighted by the red line (after Nakata and Imaizumi 2002). The broken blue line roughly indicates the currently inactive part of the ISTL. The lower panels show the estimated directions of the principal stress axes (stereo net) and the estimated value of stress ratio $\phi$ (histogram) obtained in the circled area (after Imanishi et al. 2010)

time 22:08 (UTC + 9) with an estimated local magnitude $\left(M_{\text {JMA }}\right)$ of 6.7 . This earthquake created a prominent surface offset up to $1 \mathrm{~m}$ (Figs. 2, 3) spanning an area of $9 \mathrm{~km}$ which generally coincided to a part of the previously mapped Kamishiro fault segment of the ISTL (Okada et al. 2015), indicated by the red line in Fig. 1. The F-net centroid moment tensor (CMT) solution catalog (http:// www.fnet.bosai.go.jp/top.php?LANG=en) by National Research Institute for Earth Science and Disaster Prevention (NIED) confirmed the general motion of the Kamishiro fault and showed it to be an east-southeast dipping reverse fault that also contains a considerable non-double-couple component (Fig. 4).

Importantly, the crustal deformation associated with this inland earthquake was captured by the synthetic aperture radar (SAR) imagery over the entire region (Fig. 3); the analyzed result of the interferometric synthetic aperture radar (InSAR) is presented here for the observation pair of October 2, 2014, and November 27,2014 . The striking feature of the surface deformations clarified by these detailed observations is that the prominent surface breaks occurred intensively on the southern half of the focal area, while only weak surface signature was identified on the northern half. Further, the InSAR image confirms that the prominent surface breaks occurred along a part of the surface trace of the Kamishiro fault (Fig. 3) as also reported based on the field surveys (Okada et al. 2015). However, this appears to be in contrast to the kinematic slip inversion results based on InSAR (Yarai et al. 2015) and by strong motion records (Asano et al. 2015), which showed that a significant amount of the fault slip actually occurred at the relatively deeper parts of the northern half of the fault rather than that of the southern part.

On the other hand, the aftershock activity (Fig. 4) indicates complex fault geometry exhibiting a significant variation along strike with the southern part forming a relatively simple and localized fault plane, while the northern part showing complicated geometry (Sakai et al. 2015; Panayotopoulos et al. 2016). While this observed geometrical difference between the north and the south is qualitatively understandable with the difference in the fault activity in the geomorphological timescale mentioned above, it is quite unclear how this geometrical complexity played roles in determining the resulting slip distribution and surface deformation.

In addition, a clear foreshock activity lasting a few days before the mainshock is also observed on an identifiable localized plane dipping down to north-northwest (Imanishi and Uchide 2017). In fact, the orientation of this fault plane shows further complexity as oriented at an oblique angle with the overall orientation of the mainshock fault plane dipping down to ESE (Fig. 4). On the other hand, it is worth mentioning that one of the nodal planes of the first-motion polarity determined by Japan Meteorological Agency (Fig. 4) is compatible with this foreshock plane, suggesting the causal relationship between the foreshocks and the mainshock. This correlation should give us the observational constraints on the part of the 


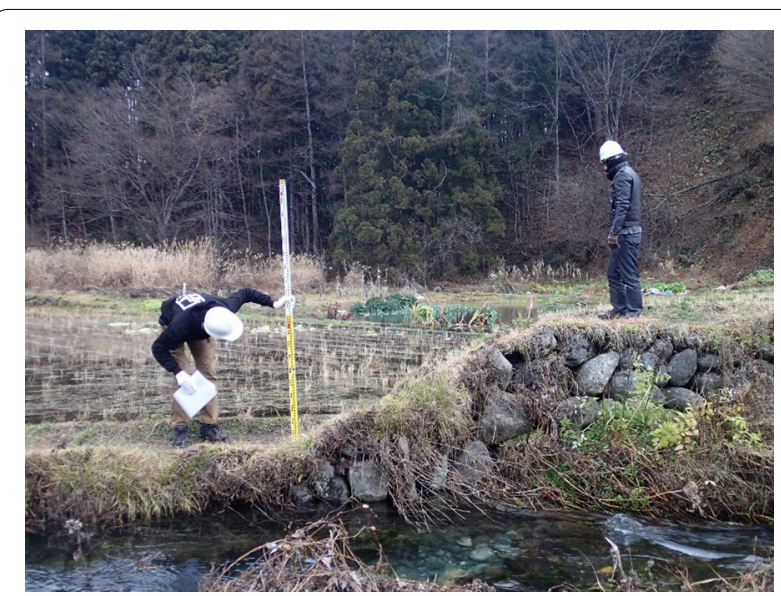

Fig. 2 An example of coseismic surface offset due to reverse faulting. Photograph taken at a site called Joyama located in the central part of the focal area. View from the south

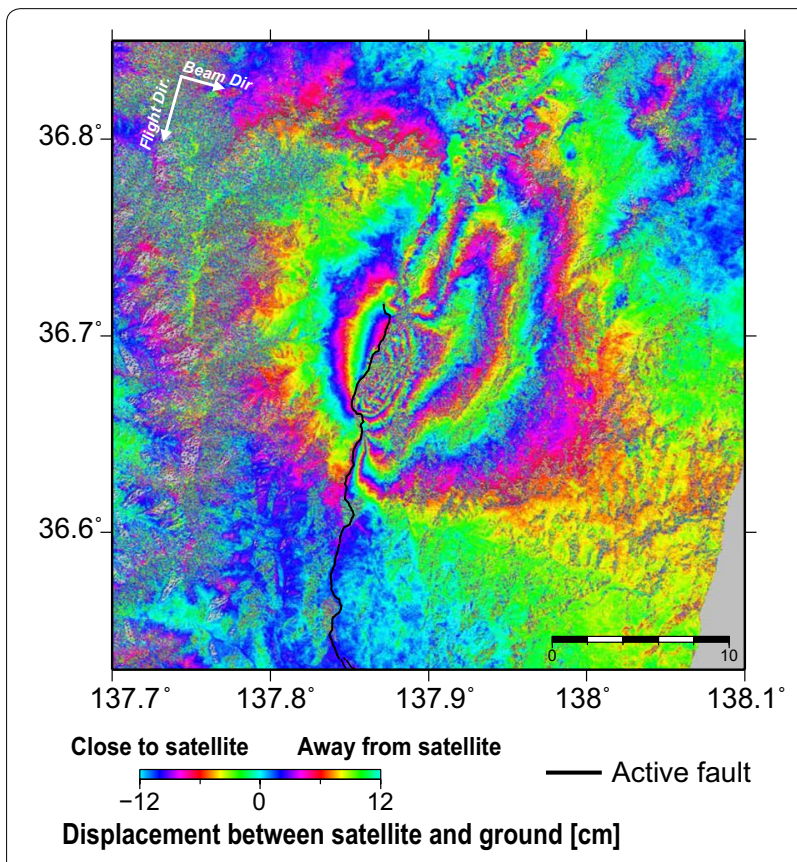

Fig. 3 Surface displacement observed after the 2014 Northern Nagano earthquake by the interferometric synthetic aperture radar. The used observation pair is October 2, 2014, and November 27, 2014. A clear surface offset is observed along parts of the previously mapped active fault trace (black line) (Sawa et al. 1999) on the southern half of the focal area

fault surface, where the nucleation occurred and initially generated the mainshock rupture.

In this study, we aim to clarify the physical mechanisms underlying the aforementioned complicated observations. In this respect, we focus on the effect of the spatially varying complexity of the fault geometry on the characteristic observed patterns in the occurrence of the foreshocks, the large difference of the first-motion focal mechanisms and the CMT and the along-strike variation of the surface displacement.

We utilize the fully dynamic 3-D rupture simulation based on the spatiotemporal boundary integral equation method (ST-BIEM) (e.g., Aochi and Fukuyama 2002; Ando and Okuyama 2010). Due to its high numerical accuracy, the ST-BIEM is an optimal numerical method able to handle the non-planar fault geometry by appropriate meshing of several complicated non-planar fault surfaces. While the ST-BIEM is a computationally demanding method having a time complexity of $O\left(N^{3}\right)$, recent development of the fast domain partitioning BIEM (FDP-BIEM) by Ando (2016) allows us to reduce it to $O\left(N^{2}\right)$. The FDP-BIEM enables realistic simulations for dynamic earthquake rupture processes.

\section{Numerical method}

The dynamic rupture problems are numerically solved by assuming the fault geometry, the on-fault stress state and the friction law as the initial and boundary conditions. It is known that fault geometry and stress state are major parameters controlling the rupture process and the resulting slip heterogeneity. Therefore, we constrained these parameters as much as possible based on the local observations in the focal area as detailed in the next section.

In FDP-BIEM, we solve the discretized boundary integral equations (BIEs) in the real space and time domain, relating the slip history with the current traction change in the form of

$$
T_{p}^{i, n}=T_{\text {init: } p}^{i}-\frac{\mu}{2 \beta} D_{p}^{i, n}+\Sigma_{i} \Sigma_{m=0}^{n-1} \Sigma_{q=1}^{3} K_{p q}^{i, j, n-m} D_{q}^{j, m},
$$

where $T_{p}^{i, n}$ denotes the $p$-th component of the traction on the $i$-th fault element at the current $n$-th time step, $T_{\text {init: } p}^{i}$ the initial traction, $D_{q}^{j, m}$ the slip rate on the $j$-th fault element at the fast $m$-th time step and $K_{p q}^{i, j, n-m}$ the integration kernel representing the elastodynamic responses at each receiver due to the slip at each source. We implement the triangular boundary elements (Tada 2006) leveraging the easiness of adapting unstructured meshing over the complicated 3-D non-planar fault geometry. The traction and slip vectors are defined on the local coordinate system defined for each element by the basis vectors $\boldsymbol{e}_{p}^{i}$, where $p=1,2$ and 3, respectively, correspond to the strike-slip, dip-slip and fault normal components. The constants $\mu$ and $\beta$ are the rigidity and the S-wave speed, respectively. The first term on the right-hand side of Eq. (1) is the instantaneous or the radiation damping term, representing the instantaneous effect of the slip on the traction change at the same element of the source and 


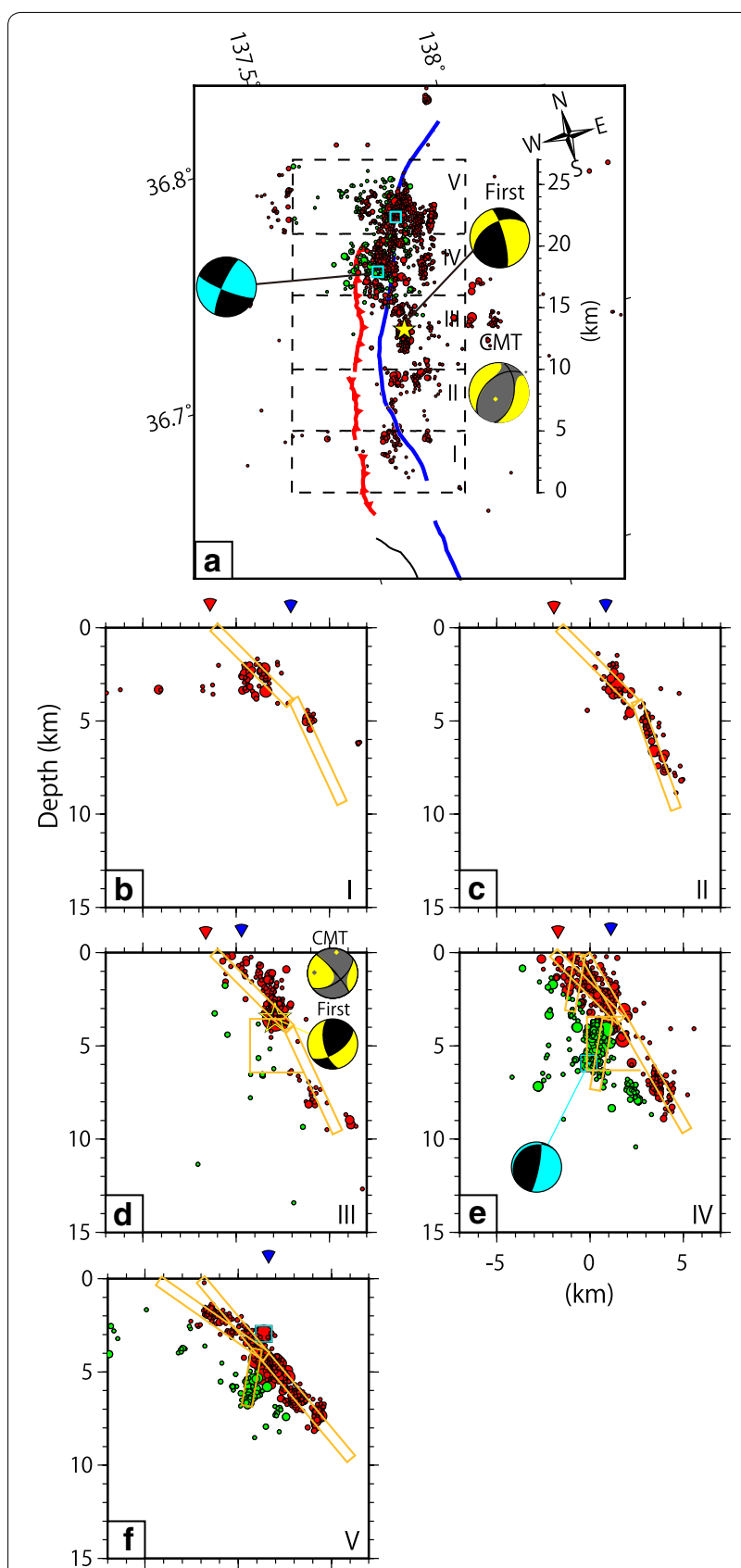

Fig. 4 Assumed fault geometry and aftershock distribution. The red and green dots, respectively, represent the hypocentral locations of the aftershocks, which are closer to the primary fault and those deviated from it, respectively (after Panayotopoulos et al. 2016). a The top view showing with the outlined fault traces (after Kato et al. 1989; Nakata and Imaizumi 2002; Kondo et al. 2008); the red and blue lines, respectively, denote surface traces of an active fault (Kamishiro fault) and a presumed geologic fault (Otari-Nakayama fault). The yellow star indicates the mainshock hypocenter. b-f The cross-sectional views for the sections I-V; the orange lines outline the cross-sectional projection of the assumed fault surfaces, and the red and blue triangles correspond to the location of the two surface fault traces. The beach balls indicate the first-motion polarity and the CMT of the mainshock (yellow) and of an off-primary fault aftershock (cyan) the receiver. We implemented FDPM for the integration on the right-hand side. The mixed boundary condition is considered where either of the shear traction $T_{p}$ or the slip rate $D_{p}$ is given for the fault surface as

$$
\left\{\begin{aligned}
D_{p}^{i, n}= & 0 \quad \text { if not ruptured } \\
T_{p}^{i, n}= & {\left[\sigma_{c o h}+\left(\mu_{s}-\mu_{d}\right)\left(S_{p}^{i, n} / D_{c}\right) T_{3}^{i, n}\right] H\left(D_{c}-S_{p}^{i, n}\right) } \\
& +\mu_{d} T_{3}^{i, n} \quad \text { if ruptured }
\end{aligned}\right.
$$

where $\sigma_{c o h}, \mu_{s}$ and $\mu_{d}$ denote the cohesional strength, the static and dynamic frictional coefficients, respectively. Here, we assumed that the frictional strength is described by the linear slip weakening friction law (e.g., Ida 1972) depending on the current slip $S_{p}^{i, n}=D_{p}^{i, n} \Delta t+S_{p}^{i, n-1}$, which gives the stress (Neumann) boundary condition. We assume no opening mode of fault displacement, giving $D_{3}^{i, n}=0$.

In addition, we mimic the elastic homogeneous and isotropic half-space, or the ground surface atop the faults, by numerically imposing a free surface boundary obeying the boundary condition of $T_{p}^{i, n}=0(p=1,2,3)$ as in Ando and Okuyama (2010). Since the mimicked free surface is bounded, we also assume an absorbing boundary condition (e.g., Cerjan et al. 1985) along the perimeter of the free surface to reduce the effect of the reflection by artificially attenuating waves. Note that, in BIEM such effects of reflection from the boundaries of finite-sized model space are significantly minor than that of volumebased methods, such as the finite element method, since the later has the boundary surfaces enclosing the volume of the model space.

As for the numerical model size, we typically employed the boundary elements of approximately 10,000 (5000 for the fault, 4000 for the ground surface and 1000 for the absorbed boundaries) as well as 1000 time steps for each rupture event. Here, the fault surface was discretized by triangular elements, with an average side length of $500 \mathrm{~m}$. This case required about $600 \mathrm{~GB}$ of memory, mostly to store the kernel values for the wave fronts, where the kernel storage to the memory greatly reduces the computation time for the convolution although FDPM works also without the memory storage (see Ando (2016) for technical details). The total computation time in this case was typically 8 min with the 36 CPUs ( 432 cores).

\section{The model}

We constrained the fault geometry model by referring the hypocentral distributions of the foreshocks and the aftershocks (Fig. 4). We mainly follow the overall fault geometry determined by Panayotopoulos et al. (2016), which describes an ESE dipping primary fault, and then, we add the secondary structures of the steps and the branches 
inferred from the hypocenters. The newly determined fault geometry is shown in Figs. 4 and 5. There, the primary fault is characterized by the left-stepping three subparallel fault segments as indicated in the surface fault mapping (Nakata and Imaizumi 2002); in Fig. 4a, the red lines within the sections I, II-III and IV almost correspond to these three fault segments, respectively, from south to north.

Regarding the secondary fault structures, one of the main features is the foreshock fault plane (hereafter, Fault F) dipping down to NNW, where we assumed the location of the mainshock hypocenter after assessing the possibility of the nucleation. Interestingly, we can observe a

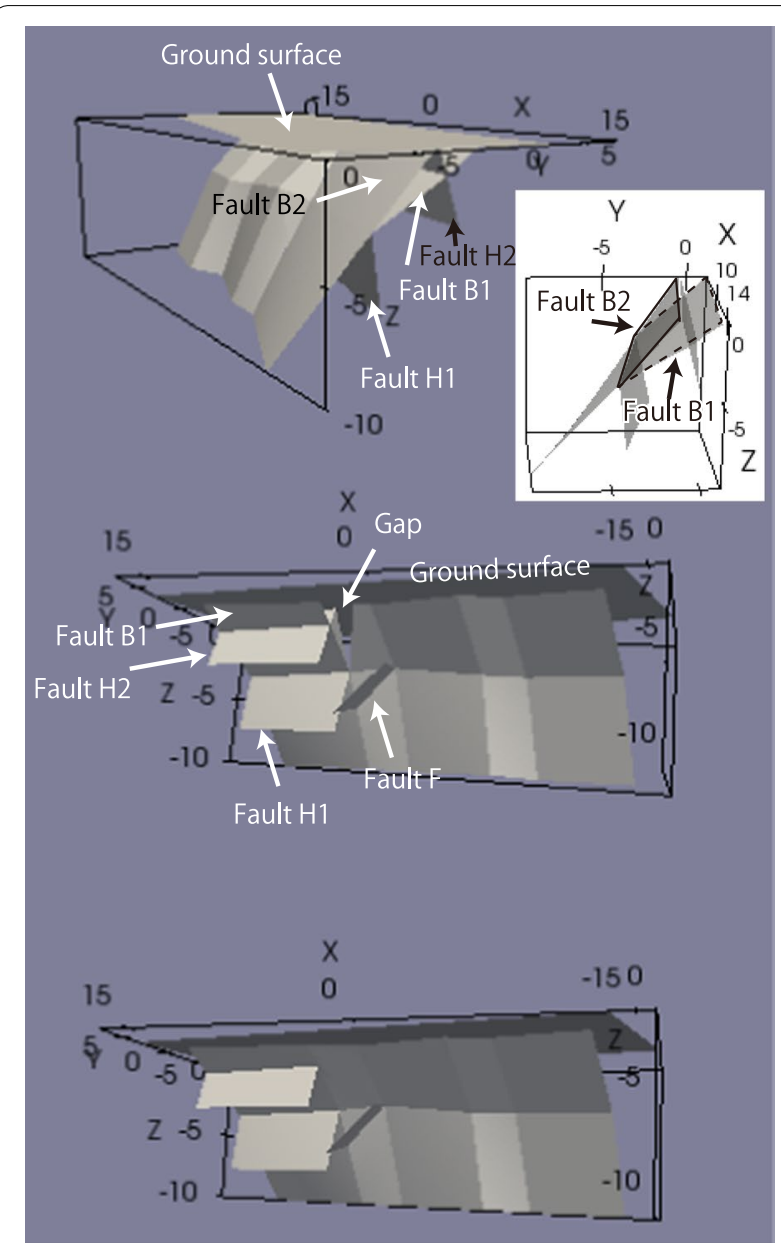

Fig. 5 Fault geometry models. View from northeast (top), looking up from southwest for Model S (middle) and for Model C (bottom). The major branch faults are, respectively, called Fault $F$ located at the center, Faults B1 and B2 for the relatively lower and higher angle ones at the shallow depth of the northern segment. The inset highlights the locations of Faults B1 and B2 enclosed by the solid and dashed lines, respectively. The back-thrust faults called Faults $\mathrm{H} 1$ and $\mathrm{H} 2$ are also assumed at the northern part. The primary fault is connected to Fault B1 in Model C by filling the gap in Model S. The X-axis is directed to north geologically identified fault trace above Fault F, where the strike of this geologic fault coincides with that of Fault $\mathrm{F}$ (Kato et al. 1989) although we do not assume the continuity each other. Other remarkable features are shallow low-angle branch fault (hereafter Fault B1) and a conjugate fault (hereafter Fault H2) assumed for the northern part, inferred from the aftershock distribution (Panayotopoulos et al. 2016) and the field surveys (Okada et al. 2015). Fault B1 is merged to Fault B2 at depth, converged to the primary fault down dip. This geometrical complexity in the north may correlate with the decreased fault activity in the north mentioned above. In addition, we assumed a prominent high-angle branch (hereafter, Fault H1) at the deeper part of the north section, where a delayed activation of strike-slip-type aftershocks was observed. The activation of Fault H1 cannot be explained in the current stress field, and therefore, the physical reason behind its current activity is of a great interest.

Rupture path selectivity and distribution or partitioning of slip to such branch faults are also an important issue in the rupture dynamics. Previous 2-D simulations have shown that the selection of the rupture paths among branch faults largely depends on the branch geometries and their relative orientations to the principal stress axes (e.g., Kame et al. 2003; Ando and Yamashita 2007). Although this condition is uniquely determined in 2-D because the faults are described by curved lines, in the current 3-D case where faults are described by curved surfaces, the condition varies depending on the rupture propagation direction. Thus, for the dominant rupture propagation directions over the northern part of the fault, we test the two different representative cases by slightly changing the fault geometry. In the first case Model S (Fig. 5 middle), we consider the mix of horizontal and upward rupture propagation, which is realized by assuming a step over (gap) between the central and northern fault segments at shallow depths. In the second one Model C (Fig. 5 bottom), we examine the case of purely horizontal rupture propagation by assuming continuous geometry and filing the aforementioned gap as a fault bend. Due to the unclear nature of the preexisting fault geometry at depth in such a small scale, we cannot predicate the relative validity of these models before conducting the simulation.

This study is designed to focus on the fault geometrical effect on the rupture dynamics; thus, the frictional effect on the rupture dynamics must be separated forehand. In order to do so, we simplify our model by assuming a homogeneous distribution of frictional parameters with $\mu_{s}=0.4, \mu_{d}=0.2, \sigma_{c o h}=0.5 \mathrm{MPa}$ and $D_{c}=40 \mathrm{~cm}$ over the entire fault. The possible effects of the spatially varied frictional strengths will be qualitatively discussed later on. 
We aim to constrain our model of the stress state by observationally based information that can be obtained prior to the event. In general, the regional stress field is inferred from the stress inversion analysis of the seismologically determined focal mechanisms by assuming the slip vectors correspond to the direction of the maximum shear traction (Michael 1987). This method enables us to infer the directions of the principal stresses $\boldsymbol{v}_{r}(r=1,2,3$ for the maximum, intermediate and minimum principal stresses, respectively) and a ratio between the principal stresses $\sigma_{r}$, defined as $\phi=\left(\sigma_{2}-\sigma_{3}\right) /\left(\sigma_{1}-\sigma_{3}\right)$. In this study, we refer the stress inversion result obtained for the targeted area of the ISTL by Imanishi et al. (2010) shown in Fig. 1, where the maximum principal stress $\sigma_{1}$ around the source region of the Northern Nagano earthquake is subhorizontal and trends approximately $\mathrm{N} 60^{\circ} \mathrm{W}$. A bootstrap test indicates that the $95 \%$ confidence region of the stress ratio $\phi$ is basically less than 0.5 , so that we assume 0.42 in the following simulation. Because these observationally constrained parameters are insufficient to determine the absolute values of the principal stresses, these remaining two unknowns are estimated by imposing the following physically and empirically acceptable assumptions. First, as often assumed (e.g., Aochi and Ulrich 2015) the vertical principal stress $\left(\sigma_{3}\right)$ is determined by the hydrostatic overburden pressure as $\sigma_{3}(h)=\left(\rho_{s}-\rho_{f}\right) g h \sim 16.7[\mathrm{MPa} / \mathrm{km}]$ from the average densities of the upper crustal rocks $\rho_{s}$ and fluid $\rho_{f}$, the gravitational acceleration $g$ and the depth $h$. Second, the other unknown given by another stress ratio $\phi^{\prime}=\sigma_{1} / \sigma_{3}$ is set to the empirically constrained value of $\phi^{\prime} \sim 1.3$. Here we consider the average stress $\operatorname{drop}\left(=\tau_{t}-\mu_{d} \tau_{n}\right)$ to be approximately $10 \mathrm{MPa}$ (e.g., Kanamori and Anderson 1975) for the nuclei location on Fault $F$, where $\tau_{t}$ and $\tau_{n}$ are the initial values of the maximum shear and normal tractions. (The compression is taken positive.) This stress model results to linearly increasing principal stresses that is supported by in situ observations estimated by deep drilling (Brudy et al. 1997).

Note that, while we apply a homogeneous regional stress field over the model space, the initial tractions on each of the fault elements are varied as a function of the fault orientation. We do that by projecting the principal stresses onto the fault surfaces as $T_{\text {init: } p}^{i}=\boldsymbol{e}_{p}^{i} \cdot\left(\boldsymbol{v}_{1} \sigma_{1}+\boldsymbol{v}_{2} \sigma_{2}+\boldsymbol{v}_{3} \sigma_{3}\right)$, where $\boldsymbol{e}_{p}^{i}$ and $\boldsymbol{v}_{q}$ are the unit vectors in the directions of $p$-th component of the traction and the $q$-th principal stress, respectively. There still exists a possibility that the regional stress field contains a small-scale heterogeneity; however, it is difficult to obtain a robust stress inversion result in a much smaller scales due to the limitation in the density of earthquake events in this region (Imanishi et al. 2010). (In a larger scale, they could conduct the stress inversion for various locations along the ISTL using earthquake clusters shown in Fig. 1.) Thus, we simply assume the homogeneous regional stress field here and focus on the on-fault traction heterogeneity due to the non-planar fault geometry.

\section{Results and discussion}

\section{Initial conditions and implications for foreshocks}

We first investigate the nucleation condition of the mainshock and its relationship with the foreshock activities. Figure 6 shows the amount of potential stress drop and the strength excess $\left(=\mu_{s} \tau_{n}-\tau_{t}\right)$, corresponding to the absolute value of the Coulomb failure stress, on the fault surface. We call the former the potential stress drop because it is the expected value once a rupture occurs, while no rupture occurred yet at this initial state. First of all, we can confirm the overall tendency of stress drop and the strength excess to increase as a function of depth, due to the depth-dependent overburden pressure. This leads to the increase of the confining and the differential stresses as depth increases. Consecutively, we can find the local variation of these values depending on the local changes in the fault orientation. A remarkable observation is that Fault $\mathrm{F}$ is oriented quite optimally although its orientation is significantly oblique in respect of the overall trend of the primary fault. In particular, the strength excess (Fig. 6b) is minimum on Fault F compared to the other parts of the primary fault at the same depth and, in addition, this fault is characterized by a reasonably large value of the potential stress drop.

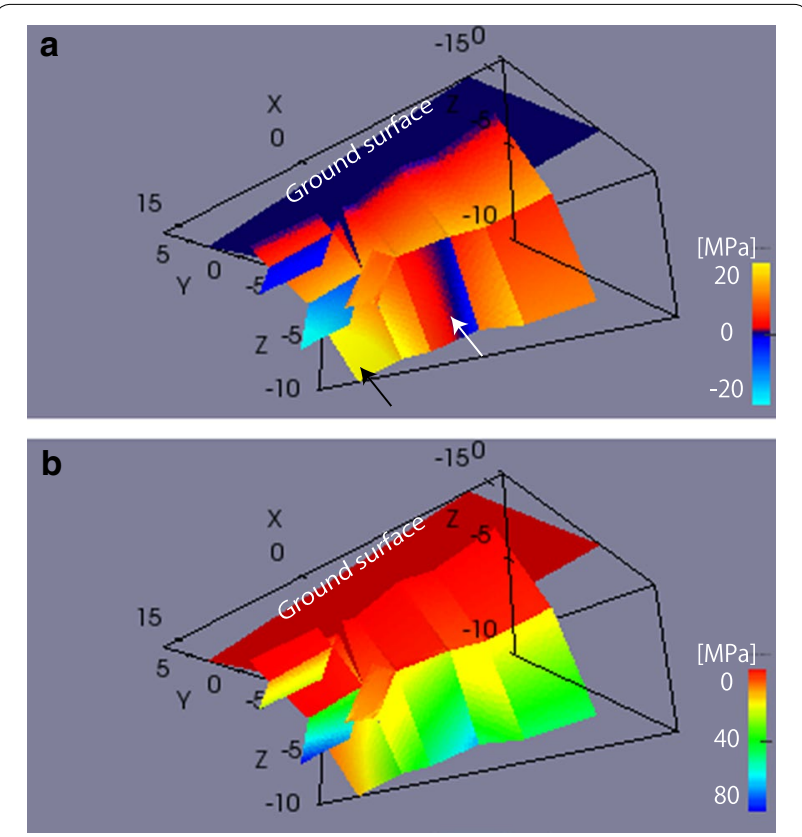

Fig. 6 Distribution of the potential stress drop (a) and the strength excess (b) for Model S. Looking up from southwest 
Another interesting observation is that the stress drop distribution exhibits its highest value on the deeper part of the north segment (denoted by the black arrow), due to the relatively lower dip angle of the fault surface. In contrast, the much smaller stress drop value on the deeper part of the central segment (denoted by the white arrow) can be attributed to the relatively higher dip angle of the fault surface; the fault surface of this part is slightly twisted, and the value is changed slightly around zero (note the discontinuous coloring). In a similar fashion, the shallower parts of the central and southern segments exhibit larger stress drop due to the relatively lower dip angles.

The aforementioned optimal orientation of Fault F may be the cause of the generation of the foreshock activity and the nucleation of the mainshock. It should be noted that we did not take into account for possible local stress perturbations due to past events or material heterogeneities. Nevertheless, we were still able to reach to a physically reasonable interpretation for the location of the precursory events and the nucleation based only on the fault geometry and the regional stress state in the current case.

\section{Rupture process}

Figure 7 shows the snapshots of the rupture processes for Model S. (A movie of the slip evolution is provided in Additional file 1: Movie S1.) The rupture initially propagates on Fault F, and as shown in Fig. 8, it increases the Coulomb stress change ( $\triangle \mathrm{CFS}$ ) on the central segment of the primary fault, and eventually the rupture jumps to the upper part of the central segment (see Fig. 7, $\Delta=1.75 \mathrm{~s}$ ). Next, the rupture expands laterally without breaking the lower part of the central segment (Fig. 7, $t=1.75-3.5 \mathrm{~s}$ ), which is signified by the negative stress drop on the lower part in Fig. 6. After rupturing the central segment, the rupture further propagates downward on the bent part to the north (positive $X$ direction) in Model S due to the existence of the gap, while the rupture keeps propagating laterally in Model $\mathrm{C}$ as recognized from the laterally continuing slip distribution shown in Fig. 9. This difference results in the after-mentioned difference in the slip distributions of the shallow part of the northern segment.

On the northern segment, the rupture initially expands on the primary fault and a branch, Fault B1, from the base of the gap (Fig. 7, $t=3.5-5.75 \mathrm{~s}$ ). (Note that the slip on Fault B1 is visible from the angle of the left panels but invisible on the right panels overlapped by Fault B2.) The rupture continues to propagate also downward along the primary fault ( $t=5.75-8.75 \mathrm{~s}$ ). The rupture of Fault B1 causes the stress shadows on the overlapped branch, Fault B2; however, since the deeper rupture expansion on the primary fault turns to increase the shear stress along the up-dip edge of the deeper section, i.e., the down-dip edge of Fault B2, eventually, Fault B2 is ruptured (Fig. 7, $t=10.5-12.25$ s., and see Additional file 1: Movie S1 for the details of timing).

We observed in Fig. 9b that the larger slip occurs on Faults B1 than B2 in both of the Models S and C. This is expected from the lower dip angle of Fault B1, which is closer to the optimal orientation. However, we find that the slip on Fault B1 in Fig. 9 appears to be larger in Model $\mathrm{C}$ than in Model $\mathrm{S}$ that is expected from the existence of the gap in Model S reducing the slip amount. Moreover, as we focus on the slip amount on Fault B2, we find that Model S displays a larger value than Model $\mathrm{C}$. This different distribution in slip on Fault B2 is explained in terms of the difference in the stress shadowing effect caused by Fault B1; the stress shadow effect appears to affect more significantly than the transient rupture velocity or the propagation directions, because there exists the time difference of each rupture, where Fault B2 is ruptured under the influence of the stress shadow caused by Fault B1. The stress shadow effect is spatially limited by the existence of the gap in Model $\mathrm{S}$ that reduces its area of influence, while it influences a larger area in Model $C$ because the slip continues to the central segment. In fact, we can observe the slip being suppressed in Model $\mathrm{C}$ on the left half of Fault B2 (Fig. 9c), while the slip on Fault B1 or on the primary fault displays a larger value (Fig. 9d). Consequently, the amount of the ground surface breaks tends to be smaller in Model S since the slip is distributed over several branches, while it becomes larger in Model C where the slip is localized to a single fault.

Note that the calculated amount of the final slip is somewhat large given the M 6.7 size of the actual earthquake event since the assumed stress drop and fault area were somewhat large. Since the slip amount is linearly scaled by the amount of the stress drop, we can obtain a more appropriate slip amount by simply reducing the stress ratio $\phi^{\prime}$, which will be better determined by trial and error without a significant influence to the simulation results.

In regard to the deeper part of the northern segment, both models allow for the propagation of the rupture and exhibit the largest amounts of slip, simply because the stress drop is largest there. The contrast between the shallow and deeper parts tends to be bigger in Model $S$ than in Model C, because of the suppression of slip in Model S from the existence of the shallow gap as well as the negative interactions between the overlapped branch Faults B1 and B2. It appears that Faults H1 on the deeper part of the northern segment was not ruptured in both Models $\mathrm{S}$ and $C$. This is understandable because the potential stress drop computed from the initial tractions shows negative values (Fig. 6). We discuss this aspect later. Similarly, Fault 


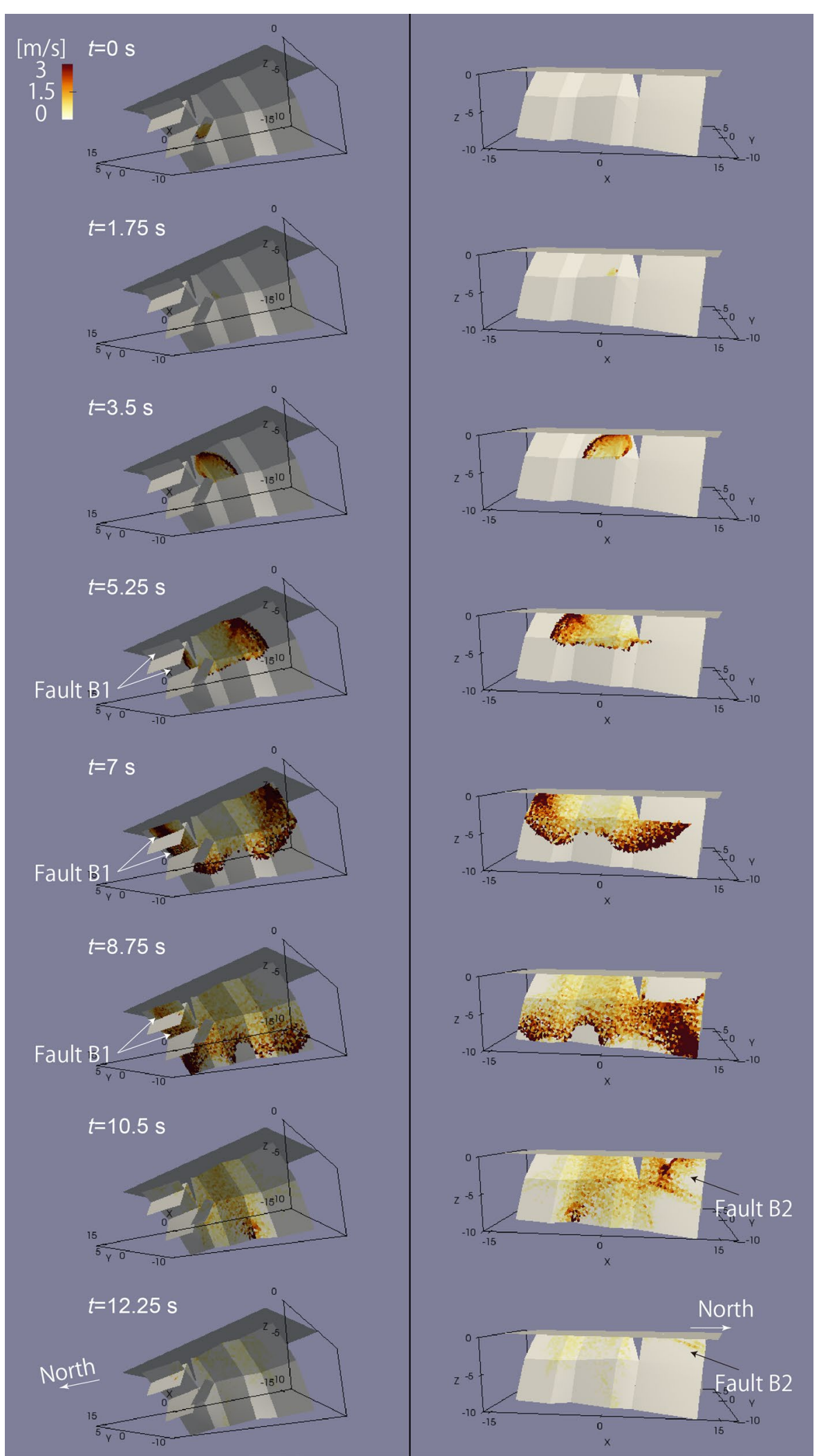

Fig. 7 Snapshots of slip rate for Models S at every $1.75 \mathrm{~s}$ from 0 to $12.25 \mathrm{~s}$. View from west (left) and from east (right) are shown. The ruptures on overlapped branch faults are marked by arrows for Faults B1 (left) and B2 (right) 


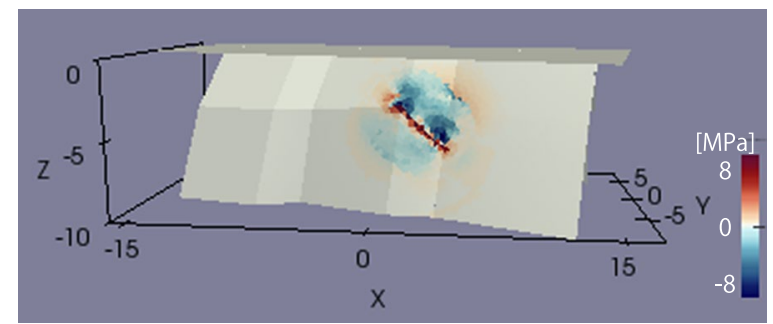

Fig. 8 Transient Coulomb stress change at $0.75 \mathrm{~s}$ after the rupture onset. Large positive values appear along the intersection to Fault $F$ located behind the primary fault. View from east

$\mathrm{H} 2$ tends not to slip, while the smaller confining pressure at the shallower depth caused small slip.

Figure 10 shows the source time functions (STFs) obtained by summing the simulated slip rates of Models $\mathrm{C}$ and $\mathrm{S}$ over the fault area. The simulated STFs show the initial peak at about $0.5 \mathrm{~s}$ corresponding to the rupture on Fault $\mathrm{F}$ and the pause at about $1 \mathrm{~s}$ corresponding to the waiting time until the rupture being transferred to the primary fault. The differences between Models $\mathrm{C}$ and $\mathrm{S}$ appear after about $3.5 \mathrm{~s}$ when the rupture encounters the gap in Model S as shown in Fig. 7. There, the rupture is delayed in propagating to Fault B1; therefore, the STF exhibits the corresponding deceleration. The STFs attain the peak values at about $7-8 \mathrm{~s}$ when the ruptures reach to the ends of the faults and they generally turn to decrease. In addition, during the decreasing phase, the STF of Model S exhibits slight acceleration around 9-10 s, while it is not significant in Model C; this acceleration actually corresponds to the delayed rupture of Fault B2.

In order to validate the dynamic characteristics of the current models, we compare the STFs with that obtained by the kinematic slip inversion analysis. Figure 10 also shows the STF inferred from the waveform inversion of the strong ground motion records, conducted by the Japan Meteorological Agency (http://www.data.jma. go.jp/svd/eqev/data/sourceprocess/event/20141122near. pdf). The plotted STFs are normalized to compare the shapes of them easily. Overall, the shapes of the largest peaks look similar to each other, but the simulation results lack the secondary peak seen in the inversion result, which is likely produced by the slip on the deeper part of the northern segment in the inversion result. In the current simulations, the gap only assumed on the shallow depth in Model S, but if the gap extended to the deeper depth as separating the northern segments from the central segment in Model S, then the timing of the slip of the deeper part of the northern segment would be delayed more significantly and would be recognized as an isolated peak. The simulated delayed rupture of Fault B2 may have additional contribution to the secondary peak.

As focusing on the increasing phase of the largest peak, the difference of the STFs between Models $C$ and $S$ is suggestive to interpret the shape of the STF in the inversion, showing deceleration at about $4 \mathrm{~s}$. While the timing and amplitude are yet to be reproduced well by the simulation, such observed deceleration may be explained by prominent geometrical irregularity such as the gap in Model S. Conversely, an assumption of smoother
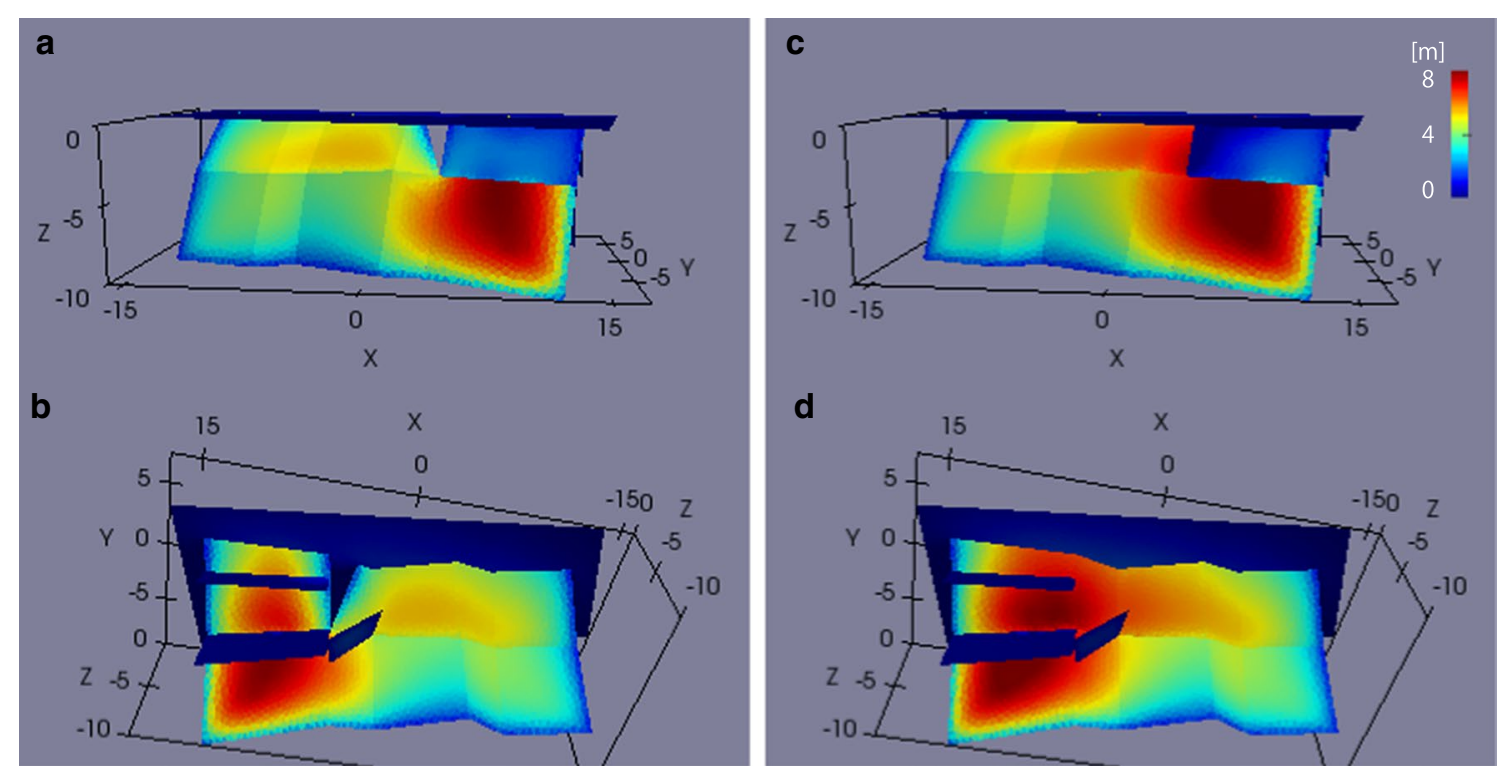

Fig. 9 Spatial distribution of final slip for Models S $(\mathbf{a}, \mathbf{b})$ and $C(\mathbf{c}, \mathbf{d})$. View from east $(\mathbf{a}, \mathbf{c})$ and looking up from west $(\mathbf{b}, \mathbf{d})$ 


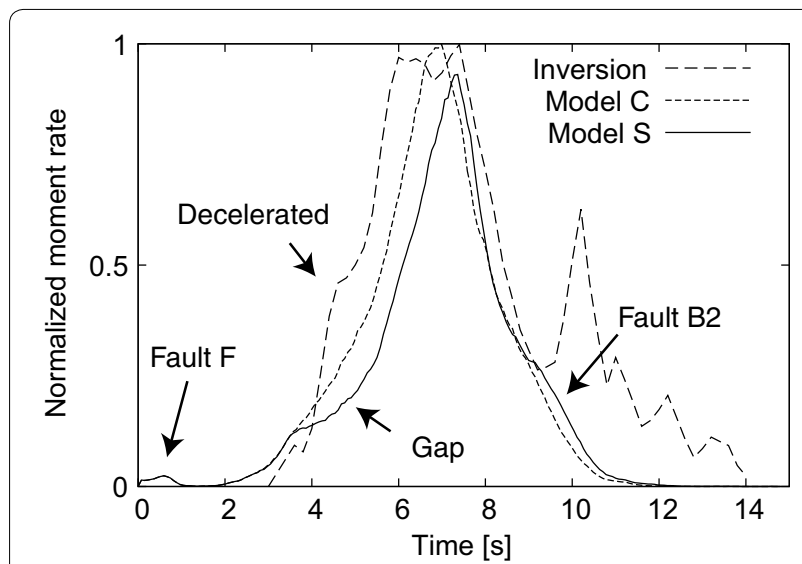

Fig. 10 Simulated and observationally inferred source time functions. Normalized moment rates are plotted for the strong motion inversion by the Japan Meteorological Agency, Model C and Model S. Both cases of Model C and Model S are normalized by the peak amplitudes of Model $C$. The inversion result is plotted $3 \mathrm{~s}$ behind the simulation results to compare their main peaks. Refer the text for details

connection between Fault $\mathrm{F}$ and the primary fault may reduce the temporal separation between the first and largest peaks in the simulations. More quantitative validations should be aimed in future studies.

Regarding the numerical resolution, the later stages of the rupture processes tend to be demanding. The minimum characteristic length scale of the current problem is defined by the size of a breakdown zone located at a rupture front, where the slip weakening process occurs. It is known that the breakdown zone shrinks as increased the rupture velocity, while the breakdown zone is sufficiently resolved by at least four boundary elements (e.g., Ando and Yamashita 2007). Both in Models C and S, the rupture velocities attain the terminal speed, the $\mathrm{S}$-wave speed, in the later stages of the rupture processes as they propagate to the deeper part of the fault. The current results hold such sufficient numerical condition in the earlier stage, but it tends to be insufficient in the later stage as the ruptures extend to the deeper part with increasing the rupture velocities. This insufficiency also causes the high-frequency numerical noise seen in the slip rate from $t=5$ to $t=6.25 \mathrm{~s}$ (Fig. 7), which is generated from the breakdown zone. However, this noise in the slip rate does not significantly affect the slip nor the STF due to its rapidly oscillating nature, and the overall rupture processes are shown to be numerically stable without numerical divergences (see also Fig. 10).

\section{Ground surface displacement}

Figure 11 shows the resulting surface displacements obtained by the simulations; the displacement

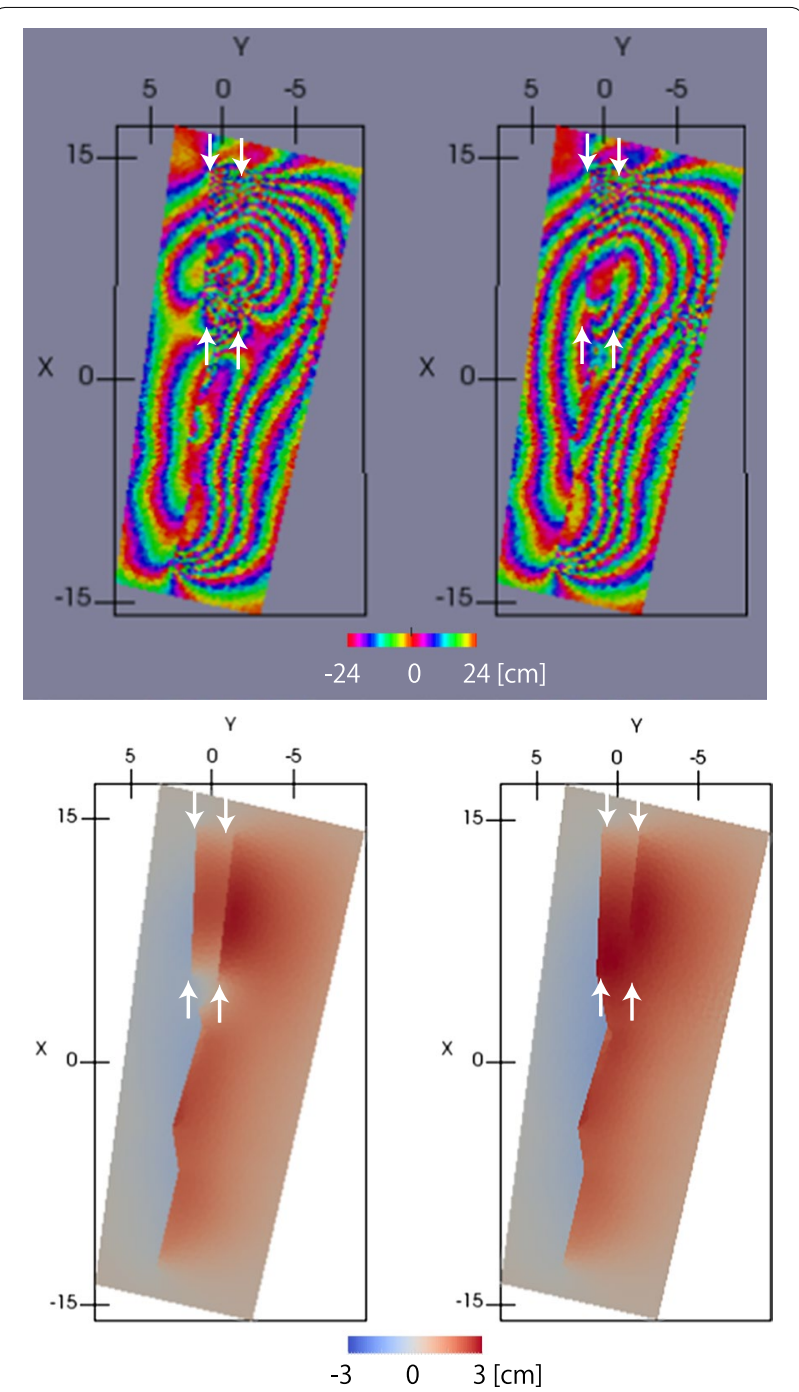

Fig. 11 Spatial distribution of surface displacement for Model S (left) and Model C (right). (Top) The fringes mimic the phases showing the line of the sight distance looking down from the west-northwest (positive $X$ - and $Y$-axis directions). (Bottom) The vertical displacement. The white arrows mark the ground surface intersections of Faults B1 and $B 2$

component is converted to the changes in displacement difference from the satellite to the surface, so that it is easily comparable to the InSAR image (Fig. 3). A prominent feature in the observed surface displacement is the significant offset along the central segment expressed by a narrow lobe of concentrated displacement (Fig. 3). This feature is well reproduced by our simulation that showed that the slip concentrated at shallow depth in that area. Another feature we were able to reproduce is a wider lobe observed in the northern part of the focal area, which reflects the deeper and larger slip on the northern segment. Conversely, the surface offsets in the northern part 
are estimated to be smaller than the southern part, something expected by the distribution of slip by the branch faults in Model S, but we were unable to reproduce the observed considerably smaller amounts of slip or the absence of surface offsets in that area in this case.

A possible mechanism to reduce the shallow slip is to simply increase the fracture energy or the placement of the barriers at the shallow depths. We did not do so in the current model by assumed a homogeneous distribution of frictional parameters for simplicity reasons, since we are mostly interested in discriminating the frictional heterogeneity effect from the geometry effect. Another possibility is to increase the number of branches in order to increase the suppression effect of rupture on the individual branches. It is difficult thus far to validate these mechanisms, but it is obvious that we cannot eliminate the later possibility from the observations of the distributed shallow aftershock activities (Fig. 4) and the abundant of the subparallel surface ruptures (Okada et al. 2015).

\section{Off-fault aftershocks and time delay}

The delayed activation of the off-fault aftershock activity probably associated with the high-angle branch fault (Fault H1) was a characteristic in the aftershock observations (Fig. 4). Figure 12 shows the Coulomb stress change $(\triangle \mathrm{CFS})$ at the static equilibrium after the dynamic rupture event. Although Fault $\mathrm{H} 1$ on the northern segment was not ruptured in both Models S and C, the computed $\triangle C F S$ gives us a clue toward understanding the mechanism of the delayed activation of Fault H1. It is observed that the transient change of $\triangle \mathrm{CFS}$ has both positive and negative values associated with the arrivals of $\mathrm{P}$ - and $\mathrm{S}$-waves. Nevertheless, the static equilibrium state exhibits positive values in its shallow part with the juncture area showing the largest values, suggesting a large stress change due to the slip on the primary fault. Note that the

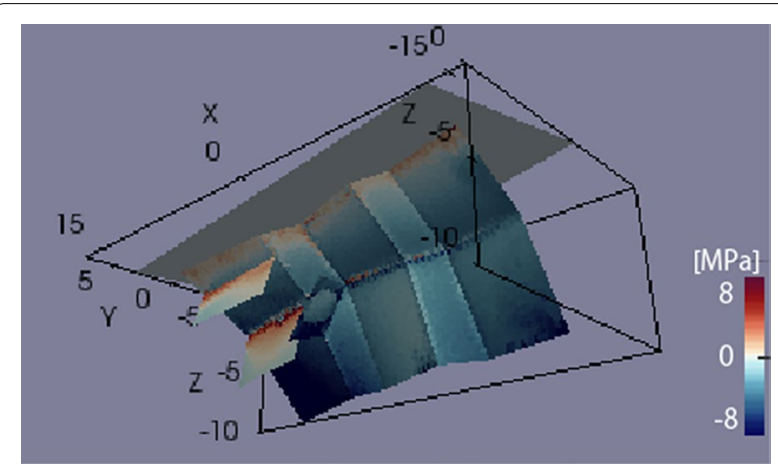

Fig. 12 Spatial distribution of Coulomb stress change for Model $C$ at the static equilibrium after the dynamic rupture event resulting amount of the stress drop at the equilibrium turns to be positive, providing the potential to generate the spontaneous ruptures, i.e., aftershocks.

Note that, due to the modeling limitations that we did not incorporate delay mechanisms in the simulation, the delayed activation of the fault slip could not be reproduced inherently. However, if we add a certain slip rate strengthening property to the friction law, we will be able to reproduce the delayed triggering as well understood in previous studies (e.g., Dieterich 1994; Nakano et al. 2010).

\section{Variation of slip direction}

Finally, the directions of the final slip are shown in Fig. 13. It is clearly observed that the overall slip direction is almost along dip $\left(90^{\circ}\right)$ on the primary fault as corresponding to the dominant component of the reverse fault motion in the observationally determined CMT. However, we can further observe a remarkable amount of the strike-slip component particularly on the bent parts of the fault where the fault is obliquely oriented against the stress axes. It is known that the non-doublecouple component is generated by the local variations of the fault geometry and slip direction, where the purely double-couple subevents alone can contaminate CMTs to have the non-double couple (e.g., Kuge et al. 1999). This suggests that the non-double component of the currently observed CMT can be explained as the geometrical effect, which is demonstrated in our simulation.

Further, the field surveys (e.g., Okada et al. 2015; Geological Survey of Japan, https://www.gsj.jp/hazards/earthquake/naganokenhokubu2014/naganokenhokubu20141126.html) discovered that the surface offset involved a considerable amount of the sinistral strike-slip component at the location where the coseismic surface rupture bends toward left, particularly near the southern end of the surface rupturing zone. This major left bend structure is also mapped previously (Sawa et al. 1999), suggesting the existence of such a persistent fault structure continuing to the certain depth. The simulated

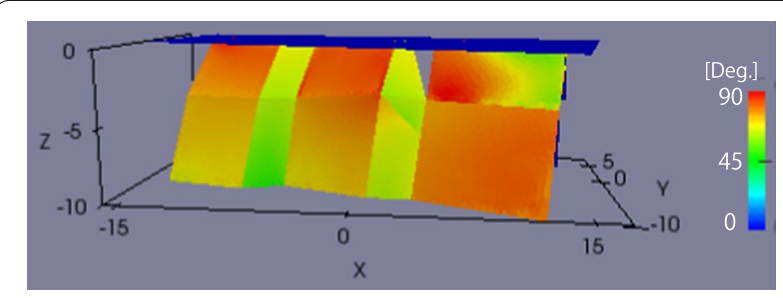

Fig. 13 Spatial variations of the slip direction for Model S. The motion of the hanging wall relative to the foot wall is shown where the reverse and sinistral motions correspond to $90^{\circ}$ and $0^{\circ}$, respectively. View from east 
spatial variation of the slip direction is compatible with this observation; we would like to emphasize that this simulation result is obtained as a quite natural outcome of the observationally constrained regional stress field and the non-planar fault geometry.

\section{Conclusions}

We investigated the origin of the complex patterns observed in the wave radiation and surface displacement of the 2014 Northern Nagano earthquake sequence. Such observations include the occurrence of the foreshocks, the large difference between the first-motion focal mechanisms and the CMT and the along-strike variation of the surface displacement. Observed aftershock distribution exhibits the more complex geometry in the northern half of the focal area, correlated with the along-strike variation of the fault activity and maturity. We conducted a set of the dynamic rupture simulations by taking into account the observationally constrained regional stress field and fault geometry. We showed that this observed complexity can be reasonably explained as the effect of the non-planar fault geometry with a number of branch faults and bends.

\section{Additional file}

Additional file 1: Movie S1. Spatiotemporal evolution of fault slip for Model S. View from east.

\section{Authors' contributions}

R. A. designed the current study, conducted the numerical simulations and mainly wrote the paper. K. I. conducted the stress tensor inversion. Y. P. determined the aftershock hypocenters. T. K. conducted the InSAR analysis. All authors discussed the results and contributed to the paper writing. All authors read and approved the final manuscript.

\section{Author details}

${ }^{1}$ Department of Earth and Planetary Science, School of Science, University of Tokyo, 7-3-1 Hongo, Bunkyo-ku, Tokyo 113-0033, Japan. ${ }^{2}$ Geological Survey of Japan, National Institute of Advanced Industrial Science and Technology, Central 7, 1-1-1 Higashi, Tsukuba, Ibaraki 305-8567, Japan. ${ }^{3}$ Earthquake Research Institute, University of Tokyo, 1-1-1 Yayoi, Bunkyo-ku, Tokyo 113-0032, Japan. ${ }^{4}$ Geospatial Information Authority of Japan, 1 Kitasato, Tsukuba, Ibaraki 305-0811, Japan. ${ }^{5}$ Present Address: Research Division for Earthquakes and Disaster Mitigation, Association for the Development of Earthquake Prediction, Chiyoda Building, 1-5-18 Sarugaku-cho, Chiyoda-ku, Tokyo 101-0064, Japan.

\section{Acknowledgements}

ALOS-2 data were provided from the Earthquake Working Group under a cooperative research contract with JAXA (Japan Aerospace Exploration Agency). The ownership of ALOS-2 data belongs to JAXA. For this study, we have used the computer systems of the Earthquake and Volcano Information Center of the Earthquake Research Institute, the University of Tokyo.

\section{Competing interests}

The authors declare that they have no competing interests.

\section{Ethics and approval and consent to participate} Not applicable.
Funding

This study is supported in part by JSPS/MEXT KAKENHI Grant Numbers JP25800253 and JP26109007.

\section{Publisher's Note}

Springer Nature remains neutral with regard to jurisdictional claims in published maps and institutional affiliations.

Received: 4 April 2017 Accepted: 5 September 2017

Published online: 18 September 2017

\section{References}

Ando R (2016) Fast Domain Partitioning Method for dynamic boundary integral equations applicable to non-planar faults dipping in 3-D elastic half-space. Geophys J Int 207:833-847. doi:10.1093/gji/ggw299

Ando R, Okuyama S (2010) Deep roots of upper plate faults and earthquake generation illuminated by volcanism. Geophys Res Lett 37:L10308. doi:10.1029/2010GL042956

Ando R, Yamashita T (2007) Effects of mesoscopic-scale fault structure on dynamic earthquake ruptures: dynamic formation of geometrical complexity of earthquake faults. J Geophys Res. doi:10.1029/2006JB004612

Aochi H, Fukuyama E (2002) Three-dimensional nonplanar simulation of the 1992 Landers earthquake. J Geophys Res. doi:10.1029/2000JB000061

Aochi H, Ulrich T (2015) A probable earthquake scenario near Istanbul determined from dynamic simulations. Bull Seismol Soc Am 105:1468-1475

Asano K, Iwata T, Kubo H (2015) Source rupture process of the 2014 Northern Nagano earthquake estimated by strong motion data. In: Japan geoscience union meeting, STT54-02, Chiba

Brudy M, Zoback MD, Fuchs K, Rummel F, Baumgartner J (1997) Estimation of the complete stress tensor to $8 \mathrm{~km}$ depth in the KTB scientific drill holes: implications for crustal strength. J Geophys Res 102:18453-18475

Cerjan C, Kosloff D, Kosloff R, Reshef M (1985) A nonreflecting boundarycondition for discrete acoustic and elastic wave-equations. Geophysics 50:705-708. doi:10.1190/1.1441945

Dieterich J (1994) A constitutive law for rate of earthquake production and its application to earthquake clustering. J Geophys Res 99:2601-2618. doi:10.1029/93jb02581

Ida Y (1972) Cohesive force across the tip of a longitudinal-shear crack and Griffith's specific surface energy. J Geophys Res 77:3796-3805

Imanishi K, Uchide T (2017) Non-self-similar source property for microforeshocks of the 2014 Mw 6.2 Northern Nagano, central Japan, earthquake. Geophys Res Lett. doi:10.1002/2017GL073018

Imanishi K, Kuwahara Y, Cho I, Hirata N, Panayotopoulos Y (2010) Present-day stress field along the Itoigawa_-Shizuoka tectonic line active fault system by microearthquake observation. In: Japan Geoscience Union Meeting, Abstract SSS017-007

Kame N, Rice JR, Dmowska R (2003) Effects of prestress state and rupture velocity on dynamic fault branching. J Geophys Res. doi:10.1029/200 2JB002189

Kanamori H, Anderson DL (1975) Theoretical basis of some empirical relations in seismology. Bull Seismol Soc Am 65:1073-1095

Kato H, Miura K, Sato T, Takizawa F (1989) Geology of the Omachi District Quadrangle Series, Scale 1:50,000. Geological Survey of Japan, Tsukuba

Kondo H, Toda S, Okumura K, Takada K, Chiba T (2008) A fault scarp in an urban area identified by LiDAR survey: a case study on the Itoigawa-Shizuoka tectonic line, central Japan. Geomorphology 101:731-739. doi:10.1016/j. geomorph.2008.02.012

Kuge K, Kikuchi M, Yamanaka Y (1999) Non-double-couple moment tensor of the March 25, 1998, Antarctic Earthquake: composite rupture of strikeslip and normal faults. Geophys Res Lett 26:3401-3404. doi:10.1029/199 9GL005420

Matsuta N, Ikeda Y, Sato H (2004) The slip-rate along the northern ItoigawaShizuoka tectonic line active fault system, central Japan. Earth Planets Space 56:1323-1330

Michael AJ (1987) Use of focal mechanisms to determine stress-a control study. J Geophys Res 92:357-368. doi:10.1029/JB092iB01 p00357 
Nakano M, Kumagai H, Toda S, Ando R, Yamashina T, Inoue H (2010) Source model of an earthquake doublet that occurred in a pull-apart basin along the Sumatran fault, Indonesia. Geophys J Int 181:141-153

Nakata T, Imaizumi T (2002) Digital active fault map of Japan. University of Tokyo Press, Tokyo

Okada S, Ishimura D, Niwa Y, Toda S (2015) The first surface-rupturing earthquake in 20 years on a HERP active fault is not characteristic: the 2014 M-W, 6.2 Nagano event along the Northern Itoigawa-Shizuoka Tectonic Line. Seism Res Lett 86:1287-1300. doi:10.1785/0220150052

Otofuji Y, Matsuda T, Nohda S (1985) Paleomagnetic evidence for the miocene counter-clockwise rotation of Northeast Japan-rifting process of the Japan arc. Earth Planet Sci Lett 75:265-277. doi:10.1016/0012-821x(85)90108-6

Panayotopoulos Y, Hirata N, Hashima A, Iwasaki T, Sakai S, Sato H (2016) Seismological evidence of an active footwall shortcut thrust in the Northern Itoigawa-Shizuoka Tectonic Line derived by the aftershock sequence of the 2014 M 6.7 Northern Nagano earthquake. Tectonophys 679:15-28. doi:10.1016/j.tecto.2016.04.019

Sakai S, Kurashimo E, Obara K, Iwasaki T, Takahashi H, Matsumoto S, Kamizono M, Okada T (2015) Urgent seismic observation for the 2014
Northern-Nagano Prefecture Earthquake and complex fault system. In: Japan Geoscience Union, Chiba

Sato H (1994) The relationship between late Cenozoic tectonic events and stress-field and basin development in northeast Japan. J Geophys Res 99:22261-22274. doi:10.1029/94jb00854

Sawa H, Togo M, Imaizumi T, Ikeda Y, Matsuta N (1999) Active fault map in urban area, Shirouma-dake. Geospatial Information Authority of Japan, Tsukuba

Scholz CH, Ando R, Shaw BE (2010) The mechanics of first order splay faulting: the strike-slip case. J Struct Geol 32:118-126. doi:10.1016/j.jsg.2009.10.007

Tada T (2006) Stress Green's functions for a constant slip rate on a triangular fault. Geophys J Int 164:653-669

Wesnousky SG (1988) Seismological and structural evolution of strike-slip faults. Nature 335:340-342

Yamaji A (1990) Rapid intraarc rifting in Miocene northeast Japan. Tectonics 9:365-378. doi:10.1029/TC009i003p00365

Yarai H, Kobayashi T, Morishita Y, Yamada S, Tobita M (2015) Crustal deformation derived from the northern Nagano prefecture earthquake detected by InSAR analysis using ALOS-2 data. In: Japan Geoscience Union Meeting, STT54-02, Chiba

\section{Submit your manuscript to a SpringerOpen ${ }^{\circ}$ journal and benefit from:}

- Convenient online submission

- Rigorous peer review

- Open access: articles freely available online

- High visibility within the field

Retaining the copyright to your article

Submit your next manuscript at $\boldsymbol{\nabla}$ springeropen.com 